See REVIEW page 15

See REVIEW page 22

See REVIEW page 31

See ARTICLE page 43

See ARTICLE page 53

\title{
To be or not to be a pathogen: that is the mucosally relevant question
}

\author{
PJ Sansonetti ${ }^{1,2,3}$
}

The human interface with the microbial world has so far largely been considered through the somewhat restrictive angle of host-pathogen interactions resulting in disease. It has consequently largely ignored the daily symbiosis with the microbiota, an ensemble of symbiotic microorganisms engaged in a commensal, and for some of them mutualistic, interaction. This microbiota heavily populates essential surfaces such as the oral and intestinal cavity, the upper respiratory tract, the vagina, and the skin. Host response to the pathogens is characterized by quick recognition combined with strong innate (i.e., inflammatory) and adaptive immune responses, causing microbial eradication often at the cost of significant tissue damage. Response to the symbiotic microbiota is characterized by a process called tolerance that encompasses a complex integration of microbial recognition and tightly controlled innate (i.e., physiological inflammation) and adaptive immune responses. This dichotomy in host response is critical at the gut mucosal surface that is massively colonized by a diverse population of bacteria. The host is therefore permanently facing the challenge of discriminating among symbiotic and pathogenic bacteria in order to offer an adapted response. This asks the fundamental existential question: "to be or not to be... a pathogen." This review has attempted to consider this question from the host angle. What do host mucosal sensing systems see in the bacteria to which they become exposed to establish proper discrimination? A new facet of medicine resides in the dysfunction of this complex balance that has likely forged the complexity of the immune system.

Our interface with the microbial world is characterized by the dual necessity to peacefully coexist with symbiotic microorganisms (i.e., the microbiota) - some of which being commensal and other being engaged in a true mutualistic relation, providing the host with vital functions-and to quickly respond to the microbial pathogens to which we are intermittently exposed. It is likely that the innate and adaptive immune systems of vertebrates have been evolutionarily "forged" by this paradoxical constraint. In humans and higher primates, the microbiota comprises a large population of diverse bacterial species present in the oral cavity, upper respiratory and digestive tract, in the vagina, and on the skin. Approximately $10^{14}$ microorganisms are present in the colon alone, a number of one order of magnitude superior to the combined number of somatic and germinal cells that compose the human body, and a metagenome comprising a global number of gene 100 times superior to the human genome. It is composed of $\sim 1,000$ species, in majority uncultivable, belonging - at homeostasis - to two largely dominant groups: the Firmicutes (Gram-positive, strictly anaerobic bacteria) and the Bacteroidetes (Gram-negative, strictly anaerobic bacteria). Other groups such as the Lactobacillaceae, the Mollicutes, and Proteobacteria (comprising aero-anaerobic Enterobacteriaceae) represent a minority, although the balance may dramatically switch in pathological conditions such as enteric infections and inflammatory bowel diseases ${ }^{1}$. Exhaustive metagenomic analysis recently unraveled the wealth of genomic diversity of the human gut microbiota. ${ }^{2}$ Its metabolic activity corresponds to an extra organ equivalent to the liver. ${ }^{3}$ It provides the host, in a mutualistic context, with the capacity to hydrolyze complex plant sugars, to synthesize vitamins, and detoxify xenobiotics. It also provides protection against pathogenic microbes by establishing a competitive barrier to their colonization/invasion of the mucosal surface, a physical barrier by strengthening the impermeability of the epithelium, and an immunological barrier by stimulating the development and maintaining the state of alert of the innate and adaptive immune systems. ${ }^{4}$ Considering the essential role of the microbiota in human evolution, possibly by allowing early human populations to survive climatic changes that

${ }^{1}$ Unité de pathogénie Microbienne Moléculaire, Institut Pasteur, Paris, France. ${ }^{2}$ Unité INSERM 786, Institut Pasteur, Paris, France. ${ }^{3}$ Chaire de Microbiologie et Maladies Infectieuses, Collège de France, Paris, France. Correspondence: PJ Sansonetti (philippe.sansonetti@pasteur.fr) or (psanson@pasteur.fr) 
led to deadly nutrition crises, one can hypothesize that under such strong selective pressure their major parameters have been established, such as dynamics of early colonization, balance of respective groups, stability along life, resilience, and restitutio ad integrum following an aggression.

It becomes increasingly clear that individual member species of the gut microbiota are not equal regarding their role in the host-microbe symbiosis. Its global and sustained outcome is tolerance, not ignorance. It is likely-although not demonstrated - that a majority of the species are not perceived by the host mucosa because they are sequestered away from the surface, largely because of their metabolic characteristics and extreme susceptibility to host defense factors, including possibly oxygen. ${ }^{5}$ On the other hand, a subgroup of bacterial species, whose size, diversity, and composition remains to be fully identified, interacts with the mucosal surface, particularly the epithelial lining that filters the signals emitted by this microbial population, and translates them into tolerogenic cascades, mostly by regulating the dynamics of innate and adaptive immune cell populations of the lamina propria. This involves the maintenance of a significant proportion of resident macrophages and dendritic cells in a situation of immaturity, and of a proper balance between regulatory T cell (Treg) lymphocytes and "inflammatory" lymphocytes such as Th1 and Th17 T cells. ${ }^{6}$ The mechanisms that establish the cross-talks and the nature of the tolerogenic signals are starting to be deciphered.

It stems from these studies that pathogens do not follow these rules. They disrupt the tolerogenic cascade and certainly impose their own signaling to the mucosa with a host response dominated by a strong innate, proinflammatory, immune response. The central question here is "how does the host discriminate between a symbiont and a pathogenic microorganism in order to adjust its level of immune response?"

The original paradigm of pathogen recognition was beautifully phrased by Medzhitov and Janeway in 1997, "Various PAMPs recognized by cognate PRRs expressed on APCs induce the expression of B7 molecules, thus signaling the presence of pathogens and allowing activation of lymphocytes specific for antigens derived from the pathogens. PRRs strategically expressed on the effector cells of the innate immune system induce the expression of corresponding sets of effector cytokines, which in turn direct the induction of the appropriate effector mechanisms in the adaptive immune response."

However, as pioneering as it could be, the "Janeway paradigm" cannot fully account for the differential sensing of commensal and pathogenic microorganisms. Both are in theory sensed by similar host-pathogen pattern recognition receptors (PRRs) engaged by pathogen-associated molecular motifs (PAMPs). PRRs and PAMPs are in a way, but understandably, misnamed and should accordingly be called microbe recognition receptors and microbe-associated molecular patterns. As a matter of fact, both symbionts and pathogens express very similar, if not identical, prokaryote-specific motifs such as peptidoglycan, lipopolysaccharide (LPS) for Gram-negative bacteria, lipopeptides, flagellin, nonmethylated DNA, which can be identified by Toll-like receptors (TLRs) ${ }^{8}$, Nod-like receptors ${ }^{9}$ - particularly
Nod 1 and Nod 2 that respectively recognize the peptidoglycan fragments muramyl-tri- and tetra-peptide diaminopimelateand a variety of scavenging receptors. ${ }^{10}$

One can envision three major-likely complementaryoptions to explain the bases of the host discriminating capacity: (i) either PAMPs from symbiotic microbes have undergone biochemical modifications that render them "stealth" to PRRs, or (ii) symbiotic microbes express dedicated effectors that actively induce the tolerogenic signals, or (iii) pathogens create, in addition to their generic sensing by PRRs, a particular level of stress that is perceived by the host as a signal of danger, thus driving its response toward inflammation with the consequence of microbial eradication.

\section{HYPOTHESIS 1}

Are PAMPs from symbiotic microorganisms less agonistic to PRRs than those of pathogenic microorganisms? Some evidences support this hypothesis. The lipid A (i.e., endotoxin) of Bacteroidetes LPS seems to be essentially tetra- or pentacylated, a form that is not agonistic - and even antagonistic - to the TLR4/CD14/MD2 complex. Conversely, the lipid A of commensal Enterobacteriaceae is largely hexacylated, thus agonistic to TLR $4 .{ }^{11}$ The former group, however, dominates the latter by 4-6 logs; thus, an overwhelming dominance of Bacteroidetes producing lipid $\mathrm{A}$ that is not sensed by the host and may even antagonize the sensing of the minority of Enterobacteriaceae. In the course of inflammatory bowel diseases such as Crohn's disease, striking deletion of the Bacteroidetes group is observed to the profit of proteobacteria, ${ }^{12}$ particularly Enterobacteriaceae that are observed in intimate binding to the epithelial surface, ${ }^{13}$ thus in a position where they are able to impose strong proinflammatory signals via their highly endotoxic lipid A. This is of course purely correlative and requires experimental validation, also considering the evidence that the intestinal epithelium is tolerized to LPS very early in life. ${ }^{14}$ Moreover, variations in lipid A acylation may be observed under the influence of environmental conditions that are not yet identified. In the oral cavity, Porphyromonas gingivalis is a commensal whose presence is increased in chronic periodontitis. This species was shown to produce a repertoire of 12 different lipid A molecules showing characteristic differences in their degree of glucosamine acylation and/or phosphorylation, and thus a repertoire of various degrees of endotoxicity that is likely to influence switches from symbiosis to acute or chronic gum inflammation. ${ }^{15}$ This concept, however, cannot be generalized. In Yersinia pestis, the plague bacillus, bacteria grown at $27^{\circ} \mathrm{C}$ express a highly endotoxic hexacylated Lipid A, whereas bacteria grown at $37^{\circ} \mathrm{C}$ express a nontoxic tetracylated lipid A. A recombinant strain constitutively expressing the $l p x L$ gene expresses a fully endotoxic hexacylated lipid A when grown at $37^{\circ} \mathrm{C}$, and appears totally avirulent in a murine model of infection. ${ }^{16}$ Therefore, based upon the previous examples, one cannot draw the simplistic conclusion that lipid A endotoxicity is associated with virulence and lack of lipid A endotoxicity with avirulence and symbiotic interactions.

A similar exercise can be attempted regarding flagellin and its recognition by TLR5. The categories of $\alpha$ - and 
$\varepsilon$-Proteobacteriaceae that comprise the Helicobacter and Campylobacter genus express flagellin molecules that are poorly agonistic to TLR5, compared with $\beta$ - and $\gamma$-Proteobacteriaceae, particularly Enterobacteriaceae. ${ }^{17}$ The amino-acid stretches accounting for the lack of signaling capacity have been identified. However, they also account for impairment in flagellar polymerization, and thus the presence of additional compensatory mutations that repair the loss of the motility phenotype that possibly occurred as a cost to avoiding TLR5 signaling. If one considers Helicobacter pylori as a symbiont (large percentage of the human population colonized, particularly in developing countries), accidentally causing diseases, the above could be a nice example of adaptation to host innate responses via the selection of mutations rendering PAMPs poor agonists of PRRs.

In summary, the "stealth" hypothesis makes sense as one of the strategies to achieve symbiosis, thus allowing the microbiota to engage into commensal or mutualistic interactions, but cannot be considered a signature of symbiotic vs. pathogenic bacteria, as some highly virulent pathogens, such as Y. pestis, use a similar strategy to achieve fulminant virulence. The repertoire of PRRs may still discriminate through the reading of complex signatures comprising different combinations of alterations in the agonistic PAMPs. This is the "bar code" hypothesis that states that a bacterium will be discriminated based on the innate immune reading of a combinatorial repertoire of PAMPs by a combination of PRRs. ${ }^{18}$ Confirmation will require further investigation regarding the gut microbiota, with the handicap that a large majority of its members are noncultivable microorganisms.

\section{HYPOTHESIS 2}

Do symbionts actively dampen innate immunity? This issue was implicitly considered in the previous paragraph with Bacteroidetes pentacylated lipid A possibly accounting for antagonistic activity on TLR4. It was addressed in works demonstrating that avirulent microorganisms actively dampen innate immune responses, and some mechanisms were suggested. An avirulent mutant of Salmonella typhimurium was shown to inhibit the nuclear factor- $\kappa \mathrm{B}(\mathrm{NF}-\kappa \mathrm{B})$ pathway by downregulating I$\kappa B$ degradation via the blocking of cullination of the $\mathrm{SCF}^{\beta \operatorname{TrCP}}$ (Ring) E3 ligase that carries out ubiquitination of I- $\kappa \mathrm{B} .{ }^{19}$ Another work demonstrated that Bacteroides tetaiotaomicron, a bona fide commensal species, downregulated the activation of NF- $\mathrm{\kappa B}$-dependent proinflammatory genes by inducing peroxisome proliferator-activated receptor- $\gamma$-dependant exclusion of RelA from the nucleus of exposed epithelial cells. ${ }^{20}$ It was also demonstrated that the probiotic species Lactobacillus casei antagonized the induction of proinflammatory genes in Shigella flexneri-invaded epithelial cells by decreasing the capacity of the cell to degrade I- $\kappa \mathrm{B} .{ }^{21}$ Altogether, these pieces of data indicate that symbionts can antagonize proinflammatory signals, although several questions remain, as stated below.

\section{What are the effector molecules that induce the anti-inflammatory cascade?}

The beginning of an answer was recently provided. Bacteroides fragilis protects animals from Helicobacter hepaticus-induced colitis. This anti-inflammatory activity requires expression of the $B$. fragilis capsular polysaccharide. Animals colonized with a polysaccharide-negative mutant of $B$. fragilis underwent severe $H$. hepaticus colonization leading to colitis. Purified polysaccharide suppresses proinflammatory interleukin-17 (IL-17) production by intestinal immune cells and protects from inflammatory disease through a functional requirement for interleukin-10-producing CD4 + cells. ${ }^{22}$ It has recently been shown that $B$. fragilis directs the development of gut Foxp3(+) Tregs. Monocolonization of germ-free animals with $B$. fragilis increases the suppressive capacity of Tregs by inducing anti-inflammatory cytokine production from Foxp3(+) T cells, and polysaccharide mediates the conversion of CD4(+) T cells into Foxp3(+) Treg cells that produce IL-10 during colonization. ${ }^{23}$ The role of the microbiota in controlling the development and activity of Tregs is emerging as a key topic in gut immunology. ${ }^{24}$ Moreover, $H$. hepaticus itself expresses an anti-inflammatory function in the murine gut, as mutants with a defective type 6 secretory system (T6SS) show increased numbers within intestinal epithelial cells (IECs). Effector(s) secreted through this T6SS that remain to be identified stimulate an anti-inflammatory gene expression profile in IECs, and CD4 + T cells from mice colonized with T6SS mutants produce increased IL-17 in response to IECs presenting $H$. hepaticus antigens. Thus, the H. hepaticus T6SS accounts for a phenotype that limits intestinal colonization and inflammation, thereby promoting proper homeostatic balance. ${ }^{25}$ More recently, short-chain fatty acids, which are produced from dietary fibers by the microbiota (both Bacteroidetes and Firmicutes), were shown to bind the G-protein-coupled receptor 43 (GPR43 or FFAR2) and to profoundly affect gut inflammatory responses. This observation provides another contextual link between diet, the gut microbial metabolism, and the control of innate/ inflammatory responses. ${ }^{26}$

\section{Are all symbiotic bacteria equal regarding anti-inflammatory capacities?}

Recent studies draw a more complicated picture. The potential proinflammatory role of $\gamma$-proteobacteria, particularly Enterobacteriaceae, has already been mentioned, and their possible deleterious role suggested when a loss of the balance between the various components of the microbiota occurs-that is, dysbiosis-and a naturally underrepresented group/species takes over. However, it seems that certain species that are represented at a fairly high density, particularly in the murine and human small intestine, have a clear proinflammatory potential. This was recently established for the segmented filamentous bacteria (SFB). This noncultivable Clostridium-related microorganism, which has already been shown to be a potent inducer of mucosal IgA, appears to have a major role in the postnatal maturation of gut immune functions, accounting alone for the maturation of T-cell functions (i.e., Th1, Th17, and Treg cells) that was thought so far to request the presence of a complex microbiota. ${ }^{27}$ Another publication confirmed this concept, showing that SFB was sufficient to induce the appearance of CD4 T cells producing IL-17 and IL-22 (Th17 cells) in the murine lamina propria. Colonization with SFB was correlated with increased 
expression of proinflammatory and antimicrobial defenses genes and resulted in enhanced resistance to the intestinal pathogen Citrobacter rodentium. ${ }^{28} \mathrm{SFB}$ adheres tightly to the surface of epithelial cells in the ileum of mice, a characteristic that appears rather specific to this microorganism, thereby possibly explaining its strong stimulatory effect on mucosal innate immune responses. A new paradigm is therefore emerging, according to which a limited group of species present in the microbiota essentially account for the impact on the innate response network, and the resultant of these antagonistic vectors establishing the balance that leads to tolerance while maintaining a level of readiness-that is, physiological inflammation-allowing quick and adapted response to an infectious stress. ${ }^{6}$ This renders the definition of pathogens vs. symbionts quite complicated, as the latter can now be considered forming a spectrum from totally harmless to "reasonably" virulent microorganisms. The gut microbiota so far seems to respond to a combination of hypotheses 1 and 2. In spite of the fact that most of the associations are symbiotic, commensal, or mutualistic, some members of the microbiota have the potential to cause disease, in case of host immunological failure, or major dysbiosis, and thus the term of "pathobiont" was recently coined regarding H. hepaticus ${ }^{25}$ and may as well apply to SFB. It is likely that its capacity to stimulate the proinflammatory component of the mucosal innate response can largely be attributed to its intimate binding capacity to the epithelial apex. This is an excellent introduction to the definition of pathogens that, unlike most of the commensal microorganisms, show a capacity to strongly interact with host cells and tissues.

In summary, symbiotic bacteria in interaction with the host intestinal mucosa cannot be seen only through the characterization of the pro- and anti-inflammatory capacities of their PAMPs and other effectors. One also needs a three-dimensional vision of their localization in the gut. This is likely to be a relevant characteristic in comparison with pathogens. A general view is that a large part of the microbiota resides, as a complex community, at the surface of the mucus layer that protects the epithelium from unwanted close encounters. The mucus layer serves as matrix in which antimicrobial factors such as cationic antimicrobial peptides produced by the epithelial lining are embedded. It therefore constitutes a buffer zone that not only achieves lumenal compartmentalization of the microbiota, but also establishes a communication line through which the microbiota and the epithelium can exchange molecules that support the crosstalks. Some of these bacteria can venture deeper in this buffer zone, and ultimately (SFB) establish close interaction with the epithelial apex, thereby enhancing the level of the cross-talk as mentioned before. Otherwise, it is likely that through this buffer zone, bacteria can be perceived in quantity and activity through their capacity to release PAMPs (i.e., LPS, muropeptides, flagellin), and other markers of proliferation such as quorum-sensing autoinducer molecules. Regarding LPS, its signaling capacity may be somewhat attenuated by three factors: (i) TLR4 and coactivation molecules are poorly expressed at the level of the surface epithelium, (ii) the intestinal epithelium is tolerized to lipid A early in life as mentioned above, and (iii) interestingly, it was also shown that alkaline phosphatase expressed on the apical surface of IECs in the zebrafish was able to dephosphorylate, and thus inactivate luminal lipid A. ${ }^{29}$ Muramyl-tri/ tetrapeptide diaminopimelate, which is released as a turnover by-product of permanent cell-wall biosynthesis, is perceived by intracellular Nod 1 that is constitutively expressed by IECs. Recent evidence indicates that epithelial transporters like hPepT1 may capture and transport muropeptides from the outside milieu to the cytoplasmic compartment, thereby allowing a somewhat controlled sampling of the cellular environment. ${ }^{30}$ Similarly, bacterial quorum-sensing autoinducers can be transported inside cells, thus offering the possibility to monitor the replicating activity of bacterial populations, as demonstrated for Bacillus subtilis quorum-sensing molecule CSF (competence and sporulation factor) and the IEC apical transporter OCTN2 (organic/carnitine cation transporter 2). ${ }^{31}$ Any overgrowth and excessive proximity will lead to an increased concentration of these typical prokaryotic compounds that have been harnessed in the eukaryotic signaling network, and may reach a certain threshold from which epithelial cells alter their response and pass from a tolerogenic to a somewhat more inflammatory program. This remains, however, within homeostatic limits, illustrating the concept of "physiological inflammation" that was coined earlier in this review. This concept is illustrated in Figures 1 and 2.

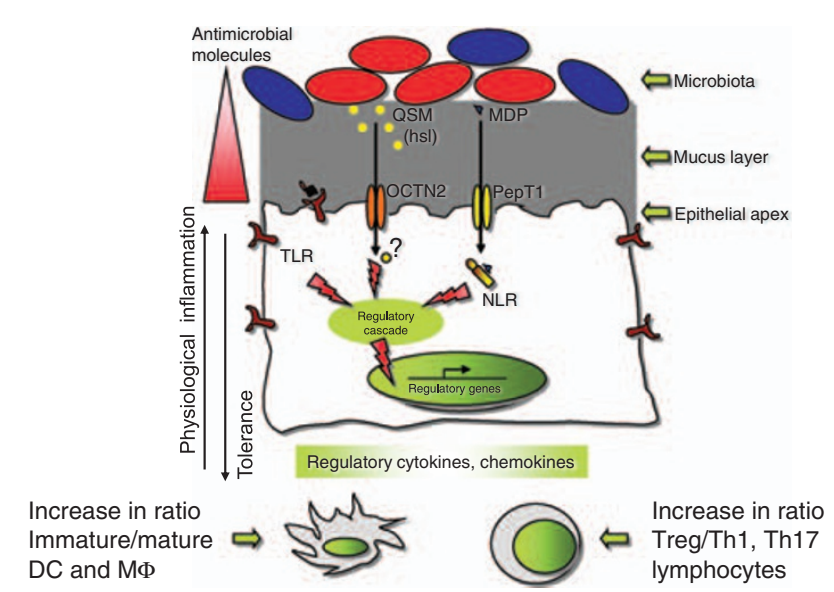

Figure 1 Mechanisms of gut homeostasis established between the symbiotic microbiota and the epithelium. In the absence of virulence factors, the microbiota is compartmentalized into the lumen, in part as a complex biofilm embedded in the soft upper portion of the mucus layer. It is kept in check, away from the epithelial surface, by antimicrobial molecules embedded in the mucus layer. Controlled amounts of bacterial pathogen-associated molecular motifs (PAMPs) and equivalent molecules such as quorum-sensing autoinducers (QSMs) are sampled by epithelial cells via apical transporters (i.e., PepT1, a proton-dependant transporter of oligopeptides, ${ }^{30}$ and OCTN2, a Na+-dependant transporter of carnitine ${ }^{31}$ ), and induce a pattern of epithelial expression that maintains a significant amount of $T$ regulatory (Treg) cells in the lamina propria and immature antigen-presenting cells (i.e., macrophages and dendritic cells (DCs)). NLR, Nod-like receptor; TLR, Toll-like receptor. MDP, muramyl dipeptide; hsl, homoserine lactone. 


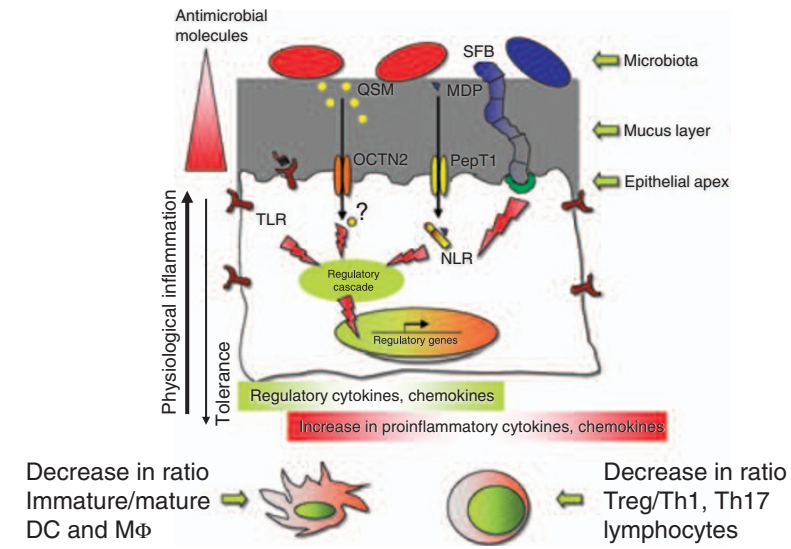

Figure 2 Components of the gut microbiota are not equal. A small percentage corresponds to "pathobionts" such as segmented filamentous bacteria (SFB) and Helicobacter hepaticus. These bacteria affect more intimate contact with the epithelial surface and express effector molecules, thus causing the induction of Th1 and Th17 "proinflammatory" lymphocytes. As part of a balanced microbiota, they are likely to maintain a level of "physiological inflammation" that supports a state of readiness of the gut mucosal immune system. In excess in an unbalanced microbiota, such "pathobionts" create a situation of dysbiosis marked by uncontrolled inflammation. DC, dendritic cell; MDP, muramyl dipeptide; NLR, Nod-like receptor; OCTN2, a Na+-dependant transporter of carnitine; PepT1, a proton-dependant transporter of oligopeptides; QSM, quorum-sensing autoinducer; TLR, Toll-like receptor.

\section{HYPOTHESIS 3}

In spite of efforts to better delineate the limit between symbionts and pathogens and push the "Janeway paradigm" as far as possible in this reasoning, it appears that a novel paradigm is needed to better define the identity of pathogens. Here comes the concept of the "second or danger signal." Over a background of PRR-mediated recognition of PAMPs that per se cannot fully account for host discrimination between symbionts and pathogens, one needs to consider the signals related to the expression of pathogenicity itself (Figure 3). Comparative genomics has nicely confirmed that bacterial pathogens harbored additional set of genes, some of which grouped in pathogenicity islands encoding complex virulence functions such as cell adhesion, cell invasion, expression of secretory apparatus and their dedicated virulence effectors, membranolytic toxins, and so on..$^{32}$ Pathogenicity strongly affects recognition signals by engaging the so-called danger signals. These danger signals may correspond to two major groups : (i) signals emitted by the cellular structures engaged by the virulence factors, and (ii) damageassociated molecular patterns, the host molecules released as a consequence of the deleterious effect of the infectious process, some of them recognized by PRRs. Heparane sulfates, for instance, are released upon proteolytic cleavage of matrix components in response to cellular damages caused by the pathogens, and behave like endogenous substitutes of LPS recognized by TLR $4 .{ }^{33}$ This is well in line with the "danger theory" advocated by Poly Matzinger. ${ }^{34}$

These events occurring in cascade are potentially too complex to get a clear view of danger signaling. One will rather focus on

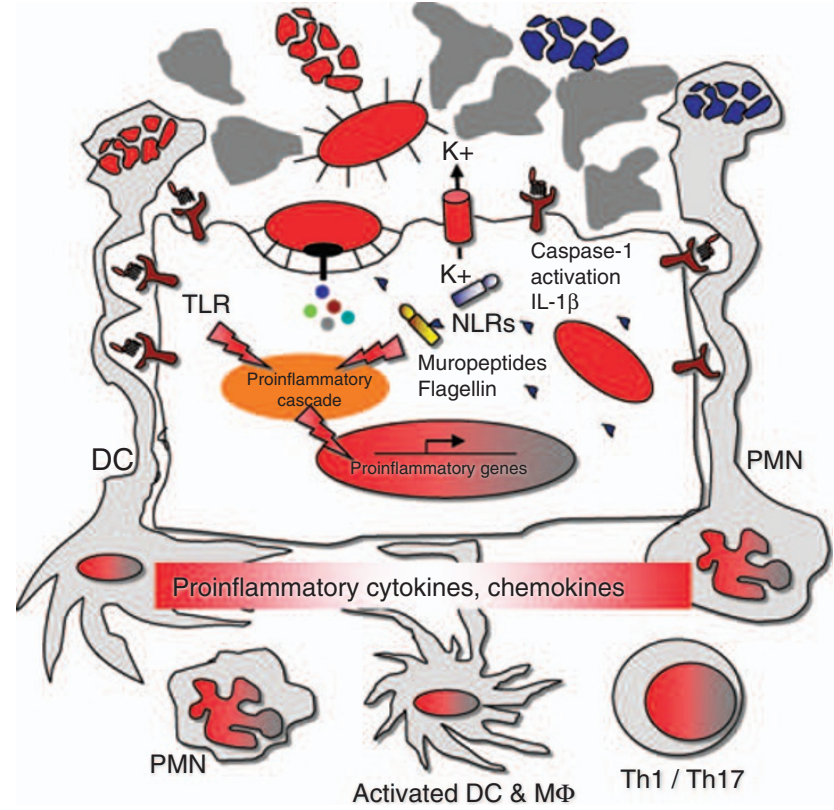

Figure 3 Pathogens have the capacity to disrupt or by-pass the homeostatic mechanisms. They can disrupt the mucus layer, attach to and/or invade epithelial cells, introduce effector molecules into the cell cytoplasm via type III or type IV secretory systems, and secrete toxins, particularly membrane-disrupting toxins (i.e., hemolysins). DC, dendritic cell; IL-1 $\beta$, interleukin-1 $\beta$; NLR, Nod-like receptor;

PMN, polymorphonuclear leukocytes; TLR, Toll-like receptor.

the former signals emitted by the cellular structures engaged by the virulence factors. ${ }^{35}$ What are the virulence factors in this context? This topic was the subject of an interesting recent review that coined the term of "patterns of pathogenesis."

Five major categories of signals can be considered:

1 - Bacterial growth inside tissues is the essence of virulence. It marks the capacity of an invading microorganism to successfully overthrow the host immune defenses, and thus to survive, multiply, and eventually disseminate in this host. Candidate molecules are many: fragments of peptidoglycan recycling such as muramyl-tri- and tetra-peptide, $30-70 \%$ of them being free in growing Gram-negative bacteria under the hydrolytic effect of the lytic transglycosylate enzyme complex that allows highly dynamic recycling of these fragments into cell-wall synthesis. ${ }^{37} \mathrm{An}$ autoinducer of quorum sensing, such as $\mathrm{N}$-(3-oxododecanoyl)L-homoserine lactone, has a significant role as chemoattractant for neutrophils, ${ }^{38}$ and similarly to formyl met-leu-phe, is a potent inducer of IL-8 expression, ${ }^{39}$ and exerts a cytotoxic effect on neutrophils, ${ }^{40}$ with part of its mode of action being possibly linked to the phosphorylation of P38 and eukaryotic initiation factor 2 -a in a TLR and Nod-independent manner. ${ }^{41}$ In addition, bacterial pyrophosphates ((E)-4-hydroxy-3-methyl-but-2-enyl pyrophosphate $)^{42}$, and di-cyclic-guanylmonophosphate ${ }^{43}$ also act as strong proinflammatory signals. It is fascinating that so many molecules reflecting bacterial growth and regulation have been harnessed by eukaryotes as signals, indicating that there are yet new families of PRRs to be discovered beyond TLRs and Nod-like receptors. 
2 - Access of bacteria to the major host barriers (i.e., to epithelial surfaces), and engagement of the cells constituting these barriers, is another source of pathogenic signals. It is likely that because of proximity and size of the inoculum, the amount of PAMPs delivered to locally available PRRs by these microorganisms trespasses the threshold set as a limit for homeostasis. The adherence process itself is a signaling source, even if the mechanisms are yet unclear. In the case of uropathogenic Escherichia coli, it was demonstrated that engagement of the membrane Gal $\alpha 1-4 \mathrm{Gal} \beta$-glycosphingolipid by the bacterial P fimbriae triggered the activation of membrane-associated acid sphyngomyelinases, leading to the production of ceramides that trigger activation of the NF- $\mathrm{KB}$ pathway and the expression of proinflammatory cytokines. ${ }^{44}$

3 - Introduction of PAMPs in the cell cytoplasm. The sampling of muropeptides via cell transporters has already been mentioned as a mean for host cells to monitor the density and proximity of its microbiota. Active introduction of PAMPs by pathogens can be seen as a much more aggressive process, bringing Nod-like receptor signaling beyond the limits of "physiological inflammation." This was initiated by the demonstration that the type III secretion apparatus-dependent Shigella led to massive, Nod1-dependent reprogramming of epithelial cells, ${ }^{45}$ and that $H$. pylori, even if extracellular, was able to cause type IV secretion apparatus-dependent introduction of muropeptides into epithelial cells, thereby also inducing Nod1-dependent induction of a proinflammatory program. ${ }^{46}$ This dichotomy is also illustrated by flagellin recognition. When delivered extracellularly, its recognition by TLR5 leads to regular PAMP-PRR activation, maintaining controlled activation of the NF- $\kappa \mathrm{B}$ signaling pathway. On the other hand, if flagellin is delivered into the cytoplasm, its recognition by Nalp5/Ipaf leads to strong activation of the inflammasome, leading to caspase- 1 activation and massive release of IL- $1 \beta$ by affected cells, particularly macrophages ${ }^{47}$ The latter situation is much more explosive, with activation of the inflammasome being clearly among the danger signals that reflect the presence of a pathogen. Broadening of this concept leads to the notion of "second signal," whereby the initial recognition of PAMPs by PRRs leads to proinflammatory priming of cells. Both commensals and pathogens are qualitatively able to induce this first phase. Conversely, only pathogens express factors are able to impose a second signal to the primed cell, such as activation of the inflammasome. ${ }^{48,49}$

4 - Entry of pathogens into cells. The invasive phenotype may be considered the ultimate threat to the host. Several signals related to bacterial invasion of cells may be considered, including the signals triggered by the bacterial effectors that induce the internalization process, and for some of them access to the cytoplasm. Salmonella, while invading polarized IECs, harness the small GTPases Cdc42 and Rac1 to regulate late events of IL-8 secretion. ${ }^{50}$ Similarly, activation of the NF- $\mathrm{\kappa B}$ pathway and IL-8 expression in IECs invaded by Yersinia is conferred by the invasin-mediated engagement of Rac $1 .{ }^{51}$

5 - Alteration of membranes. Growing evidence indicates that membrane alterations caused by virulence effectors is central to the perception of danger. Two types of effectors are particularly important. Hemolysins or membrane-lysing toxins cause membrane injury by forming pores. ${ }^{52}$ They trigger strong proinflammatory signals. Among such signals is activation of the NACHT, LRR and PYD domains containing protein 3/apoptosis-associated speck-like protein inflammasome via $\mathrm{K}^{+}$efflux. ${ }^{53}$ This concept can easily be extended to the membrane alterations caused by the formation of a transmembrane translocator by the tip structure of type III and type IV secretory systems. ${ }^{54}$ It was recently shown that in Shigella-infected epithelial cells, ubiquitinated proteins associated with vacuolar membrane remnants that recruited the autophagy marker LC3 and adaptor p62, and became targeted to autophagic degradation. In addition, inflammasome components and caspase- 1 localized to these membranes and correlated with dampened inflammatory response and necrotic cell death. These observations suggest that host membranes, following rupture by an invading bacterium, contribute to danger recognition by acting as a signaling node, with autophagy having a central role in regulating these responses. ${ }^{55}$

\section{CONCLUSION}

There have been a lot of excellent reviews addressing the definition of the genetic identity of bacterial pathogens. Most recent ones stress the dynamics of genomes in their acquisition and loss of virulence genes and gene clusters such as pathogenicity islands. ${ }^{56}$ From high-throughput genomic approaches, including microarray analysis of multiple isolates, a complex pattern emerges. Global transcriptomics also allows to identify a pattern of gene expression and loops of regulation that discriminate a pathogen such as Listeria monocytogenes from other saprophytic Listeria species. ${ }^{57}$ On the other hand, much fewer have addressed the issue with a view from the host in its permanent obligation to discriminate between a symbiotic microorganism that needs tolerance at mucosal surfaces and pathogens that need to be quickly identified and killed. It is likely that the extreme complexity of our immune system has been forged by the need to fulfill these two tasks at once. This review has shown that the "Janeway paradigm" of PAMPs being recognized by PRRs needs updating to explain this discrimination process. A second signaling step is, in general, required that is triggered by the engagement of target cells by the very factors that constitute the pathogenic identity of a virulent microorganism.

\section{ACKNOWLEDGMENTS}

P.J.S. is a Howard Hughes Medical Institute foreign scholar.

\section{DISCLOSURE}

The author declared no conflict of interest.

(C) 2011 Society for Mucosal Immunology

\section{REFERENCES}

1. Marteau, P. Bacterial flora in inflammatory bowel disease. Dig. Dis. 27, 99-103 (2009).

2. Quin, J. et al. A human gut microbial gene catalogue established by metagenomic sequencing. Nature 464, 59-65 (2010).

3. Bocci, $\vee$. The neglected organ: bacterial flora has a crucial immunostimulatory role. Perspect. Biol. Med. 35, 251-260 (1992). 
4. Bäckhed, F. et al. Host-bacterial mutualism in the human intestine. Science 307, 1915-1920 (2005).

5. Marteyn, B. et al. Modulation of Shigella virulence in response to available oxygen in vivo. Nature 465, 355-358 (2010).

6. Sansonetti, P.J. \& Medzhitov, R. Learning tolerance while fighting ignorance. Cell 183, 416-420 (2009).

7. Medzhitov, R. \& Janeway, C.A. Innate immunity: the virtues of a nonclonal system of recognition. Cell 91, 295-298 (1997).

8. Abreu, M.T. Toll-like receptor signaling in the intestinal epithelium: how bacterial recognition shapes intestinal function. Nat. Rev. Immunol. 10, 131-144 (2010).

9. Franchi, L. et al. Function of Nod-like receptors in microbial recognition and host defense. Immunol. Rev. 227, 106-128 (2009).

10. Ip, W.K et al. Mannose-binding lectin and innate immunity. Immunol. Rev. 230, 9-21 (2010).

11. Munford, R.S. \& Varley, A.W. Shield as signal: lipopolysaccharides and the evolution of immunity to gram-negative bacteria. PLoS Path. 2, e67 (2006).

12. Peterson, D.A. et al. Metagenomic approaches for defining the pathogenesis of inflammatory bowel diseases. Cell Host Microbe 3 , 417-427 (2008).

13. Swidsinski, A. et al. Mucosal flora in inflammatory bowel disease. Gastroenterology 122, 44-54 (2002).

14. Lotz, M. et al. Postnatal acquisition of endotoxin tolerance in intestinal epithelial cells. J. Exp. Med. 203, 973-984 (2006).

15. Reife, R.A. et al. Porphyromonas gingivalis lipopolysaccharide lipid $A$ heterogeneity: differential activities of tetra- and penta-acylated lipid A structures on E-selectin expression and TLR4 recognition. Cell. Microbiol. 8, 857-868 (2006).

16. Montminy, S.W. et al. Virulence factors of Yersinia pestis are overcome by a strong lipopolysaccharide response. Nat. Immunol. 7, 1066-1073 (2006).

17. Andersen-Nissen, E. et al. Evasion of Toll-like receptor 5 by flagellated bacteria. Proc. Natl. Acad. Sci. USA 28, 9247-9252 (2005).

18. Aderem, A. Phagocytosis and the inflammatory response. J. Infect. Dis. 187, S340-S345 (2003).

19. Neish, A.S. et al. Prokaryotic regulation of epithelial responses by inhibition of I-kBa ubiquitination. Science 289, 1560-1563 (2006).

20. Kelly, D. et al. Commensal anaerobic gut bacteria attenuate inflammation by regulating nuclear-cytoplasmic shuttling of PPAR-gamma and RelA. Nat. Immunol. 5, 104-112 (2004).

21. Tien, M.Y. Anti-inflammatory effect of Lactobacillus casei on Shigellainfected human intestinal epithelial cells. J. Immunol. 176, 1228-1237 (2006).

22. Mazmanian, S.K. et al. A microbial symbiosis factor prevents intestinal inflammatory disease. Nature 453, 620-625 (2008).

23. Round, J.L. \& Mazmanian, S.K. Inducible Foxp3+ regulatory T-cell development by a commensal bacterium of the intestinal microbiota. Proc. Natl. Acad. Sci. USA 107, 12204-12209 (2010).

24. Belkaid, Y., Liesenfeld, O. \& Maizels, R.M. 99th Dahlem conference on infection, inflammation and chronic inflammatory disorders: induction and control of regulatory T cells in the gastrointestinal tract: consequences for local and peripheral immune responses. Clin Exp Immunol 160, 35-41 (2010).

25. Chow, J. \& Mazmanian, S.K. A pathobiont of the microbiota balances host colonization and intestinal inflammation. Cell Host Microbe 7, 265-276 (2010).

26. Maslowski, K.M. et al. Regulation of inflammatory responses by gut microbiota and chemoattractant receptor GPR43. Nature 461, 1282-1286 (2010).

27. Gaboriau-Routhiau, V. et al. The key role of segmented filamentous bacteria in the coordinated maturation of gut helper $\mathrm{T}$ cell responses. Immunity 31, 677-689 (2009).

28. Ivanov, I.I. et al. Induction of intestinal Th17 cells by segmented filamentous bacteria. Cell 139, 485-498 (2009).

29. Bates, J.M. et al. Intestinal alkaline phosphatase detoxifies lipopolysaccharide and prevents inflammation in zebrafish in response to the gut microbiota. Cell Host Microbe 2, 371-382 (2007).

30. Vavricka, S.R. et al. hPepT1 transports muramyl dipeptide, activating $\mathrm{NF}-\mathrm{kB}$ and stimulating IL-8 secretion in human colonic Caco2/bbe cells. Gastroenterology 12, 1401-1409 (2004).
31. Fujiya, M. et al. The Bacillus subtilis quorum sensing molecule CSF contributes to intestinal homeostasis via OCTN2, a host cell membranes transporter. Cell Host Microbe 1, 299-308 (2007).

32. Dobrindt, $U$. et al. Genome dynamics and its impact on evolution of Escherichia coli. Med. Microbiol. Immunol. 199, 145-154 (2010).

33. Brunn, G.J. \& Platt, J.L. The etiology of sepsis: turned inside out. Trends Mol. Med. 12, 10-16 (2006).

34. Matzinger, P. Friendly and dangerous signals: is the tissue in control? Nat. Immunol. 8, 11-13 (2007).

35. Sansonetti, P.J. War and Peace at mucosa surfaces. Nat. Rev. Immunol. 4, 953-964 (2004).

36. Vance, R.E. et al. Patterns of pathogenesis: discrimination of pathogenic and nonpathogenic microbes by the innate immune system. Cell Host Microbe 6, 10-21 (2009).

37. Nigro, G. et al. Muramylpeptide shedding modulates cell sensing of Shigella flexneri. Cell. Microbiol. 10, 682-695 (2008).

38. Zimmermann, S. et al. Induction of neutrophil chemotaxis by the quorumsensing molecule $\mathrm{N}$-(3-oxododecanoyl)-L-homoserine lactone. Infect. Immun. 74, 5687-5692 (2006).

39. Smith, R.S. et al. IL-8 production in human lung fibroblasts and epithelial cells activated by the Pseudomonas autoinducer N-3-oxododecanoyl homoserine lactone is transcriptionally regulated by NF- $\mathrm{kB}$ and activator protein 2. J. Immunol. 167, 366-374 (2001).

40. Tateda, K. The Pseudomonas aeruginosa autoinducer N-3oxododzecanoyl homoserine lactone accelerates apoptosis in macrophages and neutrophils. Infect. Immun. 71, 5785-5793 (2003).

41. Kravchenko, V.V. et al. N-(3-oxo-acyl) homoserine lactones signal cell activation through a mechanism distinct from the canonical pathogenassociated molecular pattern recognition receptor pathways. J. Biol. Chem. 281, 28822-28830 (2006).

42. Hintz, M. et al. Identification of -4-hydroxy-3-methyl-but-2-enyl pyrophosphate as a major activator for human gammadelta T cells in Escherichia coli. FEBS Lett. 509, 317-322 (2001).

43. Karaolis, D.K. et al. Bacterial c-di-GMP is an immunostimulatory molecule. J. Immunol. 178, 2171-2181 (2007).

44. Hedlund, M. et al. Role of the ceramide-signaling pathway in cytokine responses to P-fimbriated Escherichia coli. J. Exp. Med. 183, 1037-1044 (1996).

45. Girardin, S.E. et al. CARD4/Nod1 mediates NF-kappaB and JNK activation by invasive Shigella flexneri. EMBO Rep. 2, 736-742 (2001).

46. Viala, J. et al. Nod1 responds to peptidoglycan delivered by the Helicobacter pylori cag pathogenicity island. Nat. Immunol. 5, 1166-1174 (2004).

47. Miao, E.A. et al. TLR5 and lpaf: dual sensors of bacterial flagellin in the innate immune system. Semin. Immunopathol. 29, 275-288 (2007).

48. Pétrilli, V. et al. Activation of the NALP3 inflamasomme is triggered by low intracellular potassium concentration. Cell Death Differ. 14, 1583-1589 (2007).

49. Martinon, F. et al. The inflammasomes: guardians of the body. Ann. Rev. Immunol. 27, 229-265 (2009).

50. Hobert, M.E. et al. Cdc42 and Rac1 regulate late events in Salmonella typhimurium-induced interleukin-8 secretion from polarized epithelial cells. J. Biol. Chem. 277, 51025-51032 (2002).

51. Grassi, G.A. et al. Activation of NF-kappaB and IL-8 by Yersinia enterocolitica invasin protein is conferred by engagement of Rac1 and MAP kinase cascades. Cell. Microbiol. 5, 957-971 (2003).

52. Bischofberger, M. et al. Membrane injury by pore-forming proteins. Curr. Opin. Cell. Biol. 21, 589-595 (2009).

53. Gurcel, L. et al. Caspase-1 activation of lipid metabolic pathways in response to bacterial pore-forming toxins promotes cell survival. Cell 126, 1135-1145 (2006).

54. Freche, B. et al. The role of the inflammasome in cellular responses to toxins and bacterial effectors. Semin. Immunopathol. 29, 249-260 (2007).

55. Dupont, N. et al. Shigella phagocytic vacuolar membrane remnants participate in the cellular response to pathogen invasion and are regulated by autophagy. Cell Host Microbe 6, 137-149 (2009).

56. Ahmed, N. et al. Genomic fluidity and pathogenic bacteria: applications in diagnostics, epidemiology and intervention. Nat. Rev. Microbiol. 6, 387-394 (2008).

57. Toledo-Arana, A. et al. In vivo transcriptional profiling of Listeria monocytogenes and mutagenesis identify new virulence factors involved in infection. Nature 459, 950-956 (2009). 